\title{
Mechanism of Aerodynamic Noise Generated from Front-Pillar and Door Mirror of Automobile*
}

\author{
Takahide NOUZAWA**, Ye LI**, Naohiko KASAKI** \\ and Takaki NAKAMURA** \\ ${ }^{* \star}$ Mazda Motor Corporation \\ Shinchi 3-1, Fuchu-cho, Aki-gun, Hiroshima, Japan \\ E-mail: nouzawa.t@mazda.co.jp
}

\begin{abstract}
The mechanism of aerodynamic noise generated from front-pillar and door mirror of production automobile has been investigated by applying experiment and numerical simulation. In the simulation, the flow filed was firstly analyzed by Finite Differential Method (FDM) with 3rd-order upwind scheme, then Powell's acoustic analogy was applied to evaluate the acoustic source. Furthermore, the velocity fluctuation and acoustic source were clarified by the use of experiment. It is found that the interaction between vortices separated from front-pillar and door-mirror results in the instability of boundary layer, which induces the aerodynamic noise near side window. As a result, controlling the separation vortices from front-pillar and door-mirror is the key point to reduce the aerodynamic noise of automobile.
\end{abstract}

Key words: Aerodynamic Noise, Separation, Computational Fluid Dynamics, Automobile

\section{Introduction}

Many researches including Lighthill's analogy ${ }^{(1)}$ have been performed to clarify the generation mechanism of aerodynamic noise. Powell ${ }^{(2)}$ proposed the theory that sound source is mainly induced by the variation of vortex, and Curle ${ }^{(3)}$ presented that aerodynamic noise is dominated by the pressure fluctuation on the surface of body. These theories have demonstrated that the vortex motion plays an important role on the generation of aerodynamic noise. Meanwhile, the reduction of wind noise becomes more and more important for the development of the comfortable vehicle, because the noise caused by engine, power train, tires, and other noise sources has been steadily reduced in recent years. The aerodynamic noise generated from the surrounding of side window especially gives the passenger an unpleasant feeling. Previous studies have shown that aerodynamic noise is generated by the flow separation induced by front pillar ${ }^{(4)}$ and sideview mirror ${ }^{(5)}$, but the interaction of these two separations was not discussed. In this study, we attempt to clarify the generation mechanism of aerodynamic noise induced by flow separation in front pillar and sideview mirror, where experiment and numerical simulation were applied to analyze the flow field and sound field around side window in a production vehicle.

\section{Nomenclature}

$C_{p} \quad$ : pressure coefficient

$L \quad$ : overall length of vehicle, $\mathrm{m}$ 


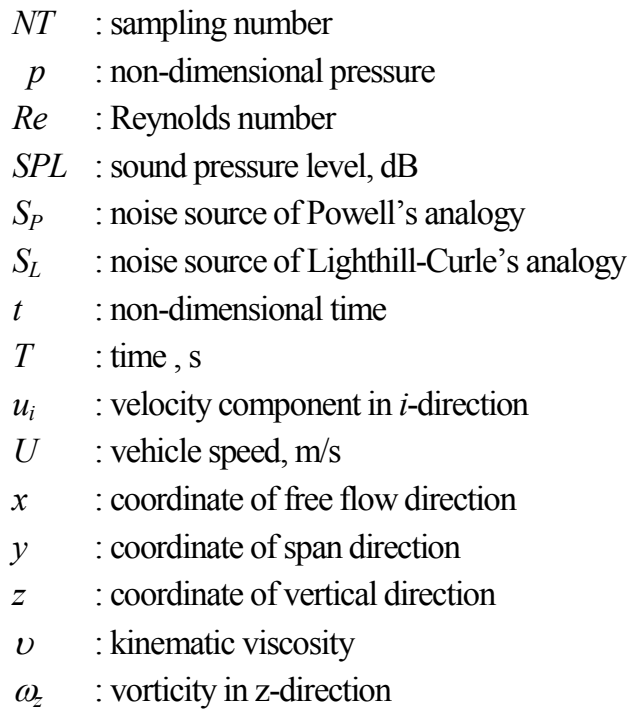

\section{Experimental Method}

In this study, a mid-size sedan type production vehicle was used, and the experiments have been conducted in Mazda Motor Corporation Wind Tunnel ${ }^{(6)}$. In order to capture the non-steady flow field, the flat microphone (B\&K 4948) was used to measure the pressure fluctuation on side window, and hot wire (I type probe) was applied to measure the time-varied velocity near side window. Moreover, the time-averaged total pressure near side window was measured by applying kernal type pressure probes. The interior noise was measured by $1 / 2$ inch condenser microphone, which is settled in the driver's ear position. For the testing conditions, the total pressure was conducted on vehicle speed of $13.9 \mathrm{~m} / \mathrm{s}$, and other measurements were $27.8 \mathrm{~m} / \mathrm{s}$. All the testing results in this paper were obtained under the condition with zero yaw angle.

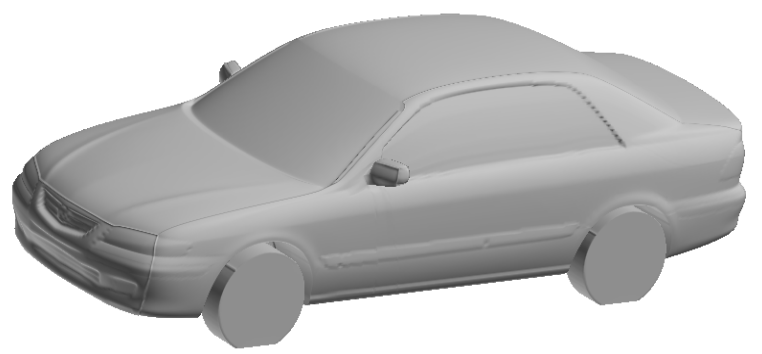

Fig.1 Production vehicle applied in current study

\section{Numerical Method}

In addition to experimental study, the numerical simulation was also conducted to analyze the flow field and noise source. Although the sound radiation and its propagation are compressible phenomenon, the sound source is dominated by the transient flow field, which can be obtained with the incompressible assumption given that Mach number $\mathrm{M}<0.1$ ( $U=27.8 \mathrm{~m} / \mathrm{s}$ ) for current study. Therefore, CFD was performed to analyze the flow field and wind noise sources, not directly calculate the radiated noise intensity because the propagation of sound wasn't considered.

The incompressible continuity equation and unsteady Navier-Stokes equations were applied as the basic equations, shown as follows. 


$$
\begin{aligned}
& \frac{\partial u_{i}}{\partial x_{i}}=0 \\
& \frac{\partial u_{i}}{\partial t}+u_{j} \frac{\partial u_{i}}{\partial x_{j}}=-\frac{\partial p}{\partial x_{i}}+\frac{1}{\operatorname{Re}} \frac{\partial^{2} u_{i}}{\partial x_{j} \partial x_{j}}
\end{aligned}
$$

Reynolds number is defined as

$$
\operatorname{Re}=U L / v
$$

where $U, L$ and $v$ represent the free stream velocity, overall length of vehicle and kinematic viscosity, respectively.

The spatial discretization is based on the finite difference method (FDM) on structured hexahedron grid. The nonlinear convective terms in Navier-Stokes equations are approximated by using following third-order upwind scheme called as K-K scheme.

$$
\begin{gathered}
\left(f \frac{\partial u}{\partial x}\right)_{k}=\frac{f_{k}\left(-u_{k+2}+8 u_{k+1}-8 u_{k-1}+u_{k-2}\right)}{12 \Delta x} \\
+\frac{\left|f_{k}\right|\left(u_{k+2}-4 u_{k+1}+6 u_{k}-4 u_{k-1}+u_{k-2}\right)}{4 \Delta x}
\end{gathered}
$$

where $f$ denotes the velocity component $u_{\mathrm{i}}$, and $k$ represents the number of grid points. The terms in the right hand of above equation include numerical viscosity, by which the small vortices smaller than grid size are diffused, and the computation becomes stable in high Reynolds number. Therefore, the approach used here can be regarded as pseudo-direct numerical simulation of turbulence. So far, this method has been widely applied for various turbulent flows, and it has been validated previously by comparing with the results obtained by experiment and LES ${ }^{(7)(8)}$.

The second-order Adams-Bashforth scheme is adopted for the time discretization in this study. For the boundary conditions, the velocity of inlet is constant, and the outlet is atmospheric pressure. The top and two sides are slip walls, and no-slip velocity condition is adopted for the surface of body and ground. To reduce the influence of boundary conditions, the computational domain is set to large size with $13 L(x$ direction $) * 10 L(y$ direction $) * 5 L(z$ direction $)$.

The total number of grids is about 10 million points for full vehicle model. Very fine grids are distributed near front pillar and sideview mirror to capture the sharp variation of flow field in these regions, where the minimum grid spacing in boundary layer is $4 \mathrm{~mm}$. The simulation was conducted in two steps. Firstly, a steady state flow field was obtained with large time step $\Delta t=0.001$. In the second step, a transient simulation was conducted with short time step $\Delta t=0.0001$ to calculate the noise source. Since this study focuses on the upper flow of vehicle, there is no engine room with the front grill shut, and only rough underbody shape is simulated in the computational model.

\section{Results and Discussion}

\subsection{Flow Field and Noise Source Around Vehicle}

The time-averaged flow field and noise source were predicted by applying CFD results. Fig. 2 shows the static pressure distribution on the surface of body, where black and white color show the negative and positive pressure region, respectively. The negative pressure is observed in the bonnet head, front bumper side, roof, front pillar and sideview mirror backward regions, where the flow is accelerated or separated. Fig. 3 shows the streamline 
near the front pillar and sideview mirror. The flow coming from the front window separates in the front pillar, and the longitudinal vortex moves toward the roof side. In addition, the wake behind sideview mirror can be observed and the flow separations in the front pillar and sideview mirror interfere it each other. The details will be described in the latter of paper.

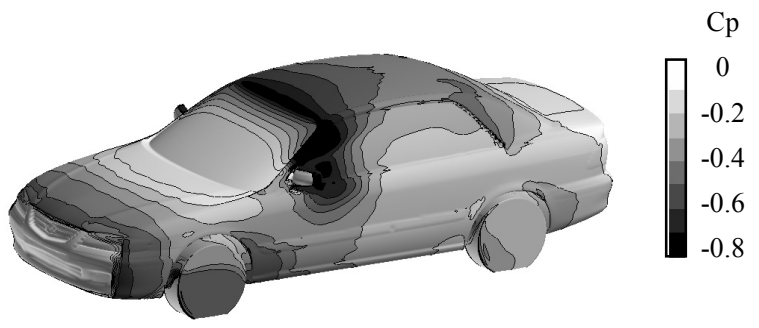

Fig.2 Static pressure on body surface (CFD result)

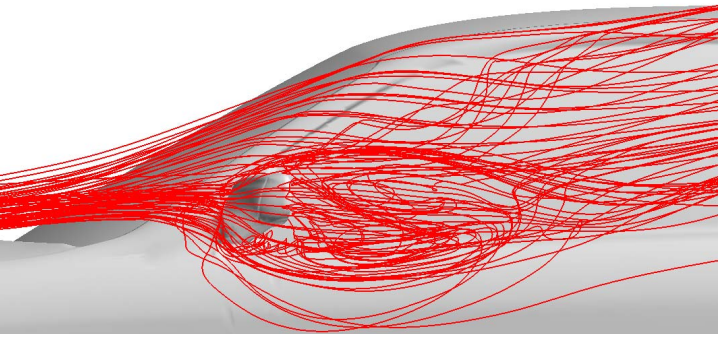

(a) Lateral View

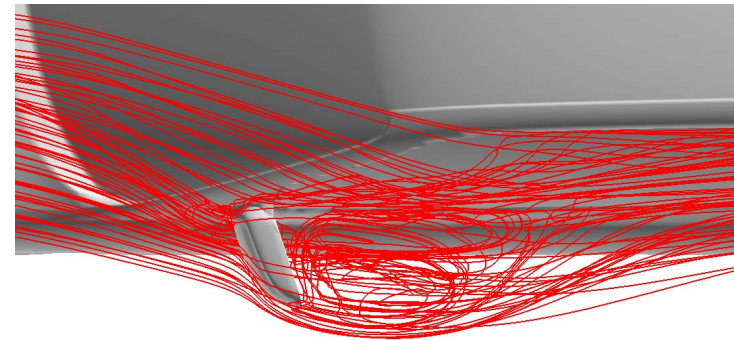

(b) Upper View

Fig.3 Flow streamlines near side window (CFD results)

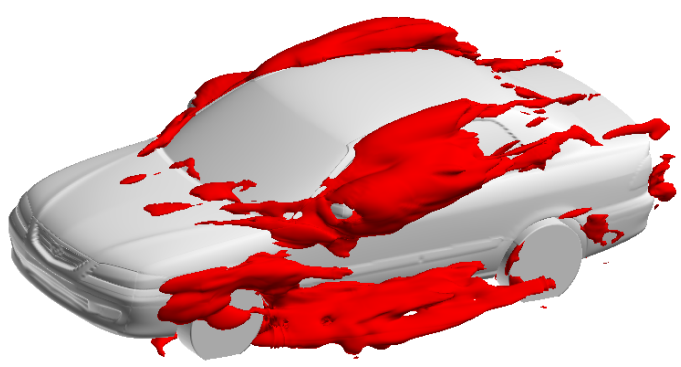

Fig. 4 Iso-surface of Lighthill-Curle's acoustic source (CFD result)

To make clear the overall image of aerodynamic noise generated from vehicle, the distribution of wind noise sources was predicted by using pressure fluctuation obtained from numerical simulation. Fig. 4 shows the structure of noise sources defined by the following Lighthill-Curle's acoustic analogy. 


$$
S_{L}=\sqrt{\sum_{i=1}^{N T}\left(d p_{i} / d t\right)^{2} / N T}
$$

where $p_{i}$ is the pressure in each time step, $N T$ represents the total computational time step and it is 10,000 in this study. The wind noise sources are mainly observed in the regions of side window and underbody in Fig. 4. It qualitatively agrees with the distribution of experimental external noise sources ${ }^{(9)}$ in these areas. However, CFD result shows weak level in bonnet head, where the noise source of this area is strong in the experiment results. The reason for this disagreement between experiment and CFD results is that the detailed shapes of front grill and bonnet head are not reproduced accurately in the CFD models, where the front grill is closed since this study focuses on the wind noise generated around side window.

\subsection{Mechanism of Aerodynamic Noise Generated From Front Pillar}

To investigate how the vortex separated from the front pillar affects the aerodynamic noise, the cross-section shape of front pillar was modified, and the flow field was analyzed. Fig. 5 shows the configuration of two models used in this study: Pillar Model A with narrow and Pillar Model B with wide section width.

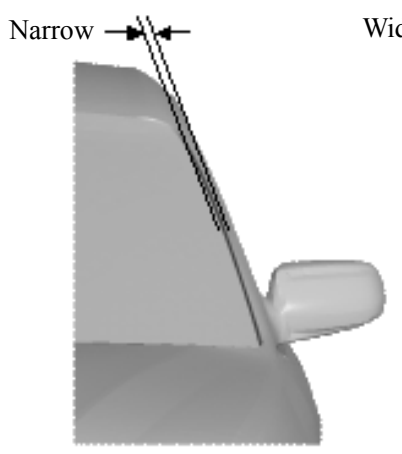

(a) Pillar Model A

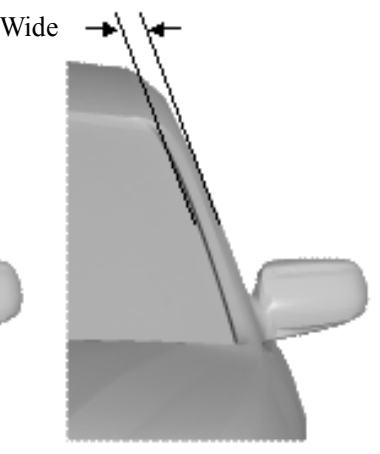

(b) Pillar Model B

Fig.5 Two models with different front pillar shape

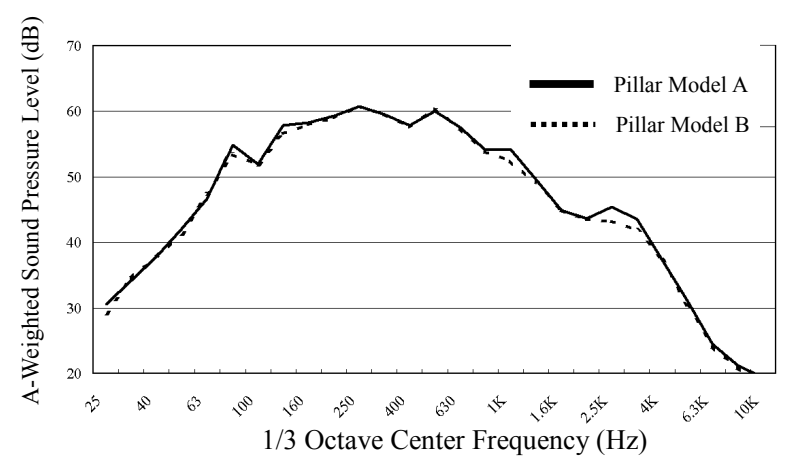

Fig.6 SPL measured in the drivers' ear position (Experiment results)

First of all, how this shape difference influences the aerodynamic noise was examined by the wind tunnel experiment. Fig. 6 shows $1 / 3$ octave analysis results of the sound measured at driver's ear position. In the case of Pillar Model B, SPL has decreased $3 \mathrm{~dB}$ in the frequency above $800 \mathrm{~Hz}$, but less hardly changes below $800 \mathrm{~Hz}$. Because the seal 
structure of vehicle is same in these two models, it suggests that the difference of this SPL is caused by the flow separation generated in the different front pillar shape.

Fig. 7 shows the total pressure distribution near the side window measured by wind tunnel experiment. The total pressure distribution divides up and down, which correspond in the flow separations from the front pillar and sideview mirror respectively. In comparison with Pillar Model A, the flow separation area (A region) behind the front pillar in Pillar Model B becomes small.

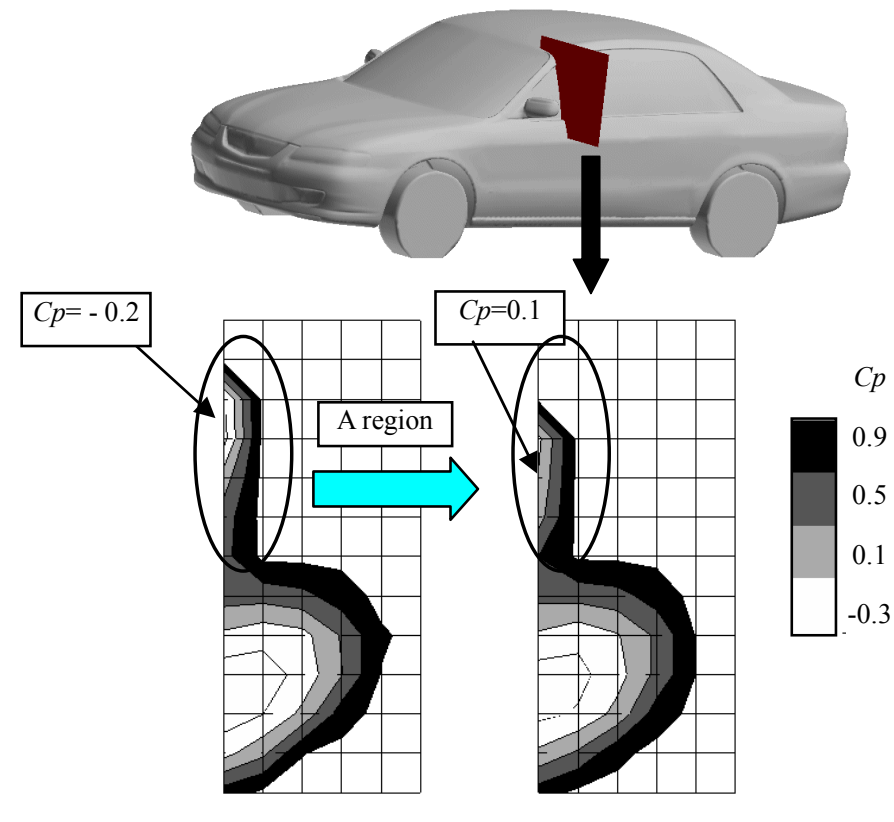

(a) Pillar Model A

(b) Pillar Model B

Fig. 7 Total pressure distribution behind front-pillar (Experiment results)

To examine the appearance of flow separation by front pillar in details, Fig. 8 shows the distribution of velocity vector and $z$ direction vorticity $\omega_{z}$ in the $x y$ section obtained by CFD. It is clear that the flow is separated from the head of front pillar. The shear layer with strong vorticity is formed in the boundary between the main flow and the separation region. Moreover, the flow separation area in Pillar Model B has become smaller than that of Pillar Model A. That shows that the flow separation is controlled by the change of front pillar shape, and the maximum vorticity of shear layer has become small as shown in Fig. 8. It is demonstrated that quadruple acoustic source exists in this area, because the velocity fluctuation and the time variation of vorticity can be observed.

Next, Fig. 9 shows the distribution of pressure fluctuation on the side window measured by experiment in order to investigate the relation between the flow field and acoustic noise, where the overall value of pressure fluctuation from 500 to $8 \mathrm{kHz}$ is indicated. The pressure fluctuation is large in the regions behind the front pillar and sideview mirror. In this area, the strong aerodynamic noise is generated from the dipole acoustic source induced by pressure fluctuation. However, comparison of the results for two models shows that the pressure fluctuation of Pillar Model B is smaller in the region behind front pillar (A region in Fig. 9). The time variation of pressure fluctuation on the surface of vehicle plays an important role on the generation of aerodynamic noise from the Lighthill-Curle's acoustic analogy. Accordingly, the results of Fig. 9 can explain why the aerodynamic noise in high frequency decreased in Pillar Model B, which was shown in Fig. 6.

By summarizing the results so far, the generation mechanism of aerodynamic noise from front pillar can be interpreted as follows: the quadruple acoustic source is generated in the 
space near side window by the vorticity fluctuation induced by the flow separation in front pillar. Moreover, the dipole acoustic source is generated on the side window by pressure fluctuation that is caused as a result of flow separation in front pillar.

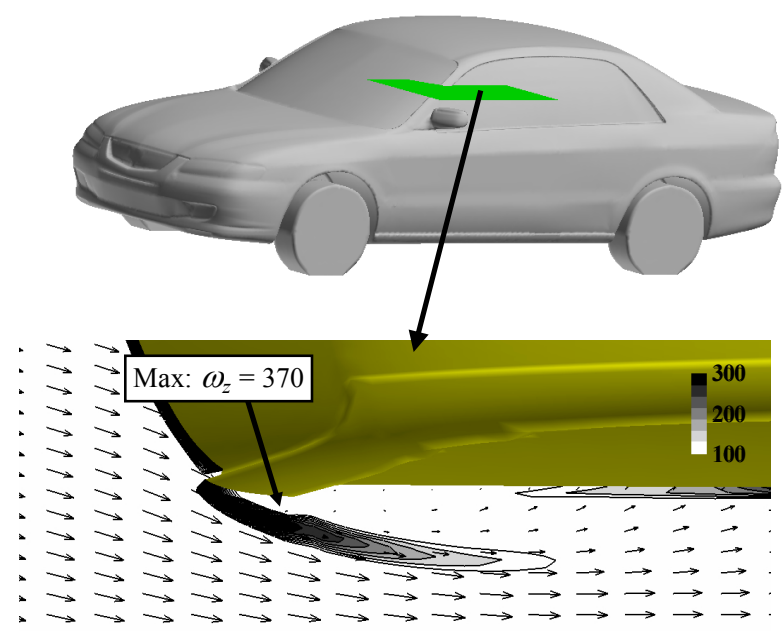

(a) Pillar Model A

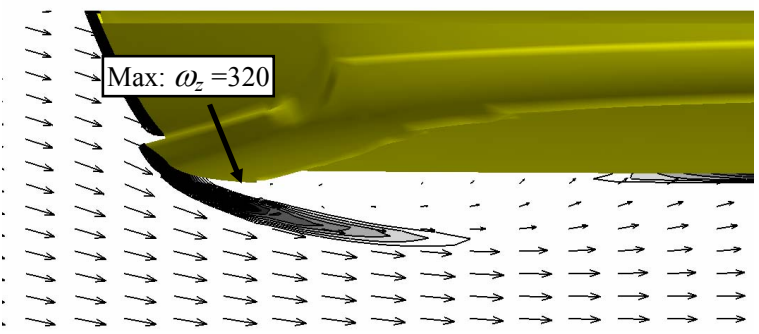

(b) Pillar Model B

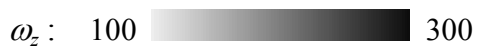

Fig.8 Distribution of velocity vector and vorticity $\omega_{z}$ (color) in the $x y$ section (CFD results)

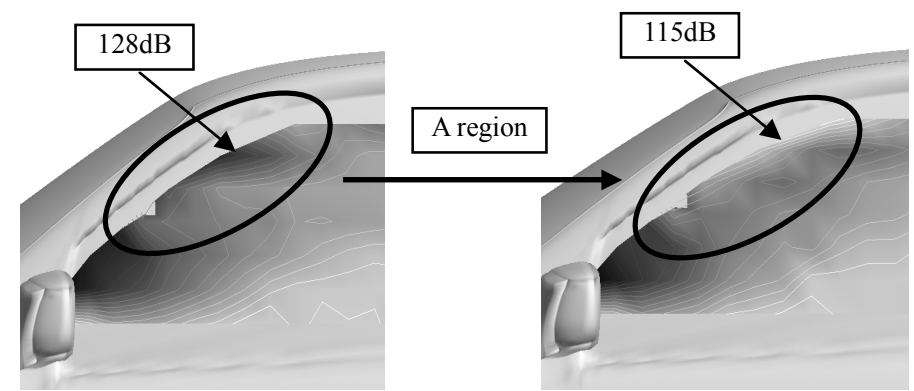

(a) Pillar Model A

(b) Pillar Model B

$115 \mathrm{~dB}$

$130 \mathrm{~dB}$

Fig.9 Intensity of acoustic source on side window (Experiment results)

\subsection{Mechanism of Aerodynamic Noise Generated From Sideview Mirror}

To investigate the relation between aerodynamic noise and flow field behind sideview mirror, two models (Mirror Model A and Mirror Model B in Fig. 10) with different position 
were examined with same vehicle shape including front pillar. Fig. 11 shows the 1/3 octave analysis result of wind noise measured at the driver's ear position. The wind noise at the ear position has decreased in the maximum by $2 \mathrm{~dB}$ for the frequency over $1 \mathrm{kHz}$ in Mirror Model B. To clarify the mechanism of aerodynamic noise reduction, the flow field was firstly analyzed by CFD.

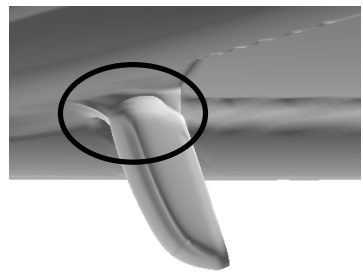

(a) Mirror Model A

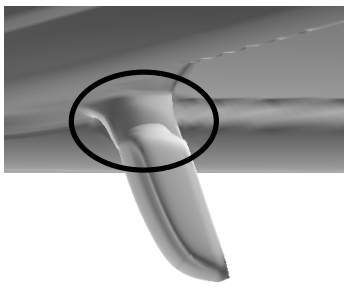

(b) Mirror Model B

Fig. 10 Two models with different door Mirror shape

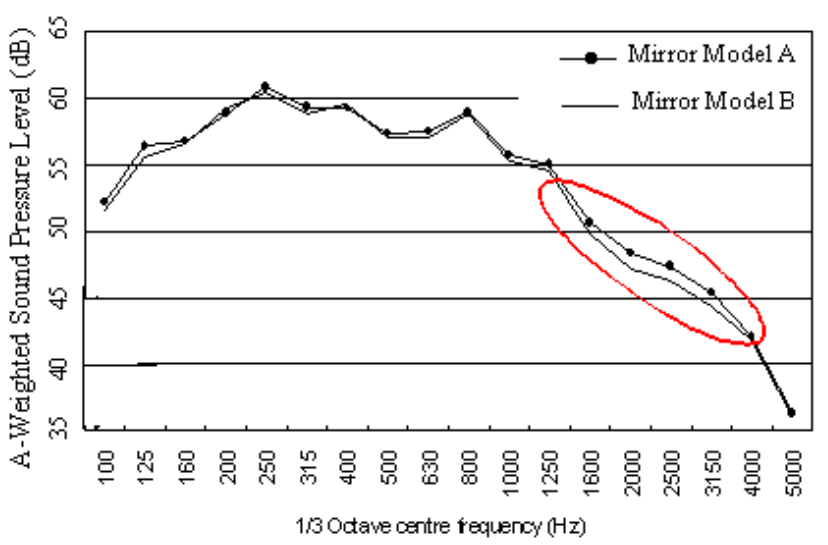

Fig.11 SPL measured in the drivers' ear position (Experiment results)

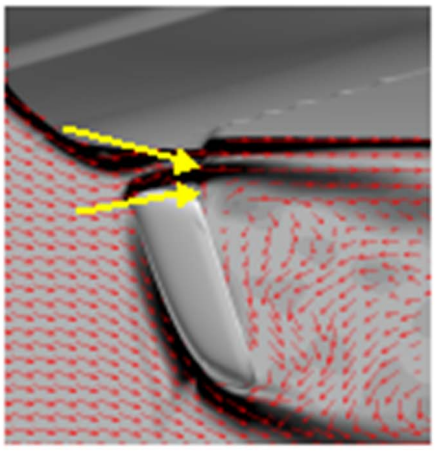

(a) Mirror Model A

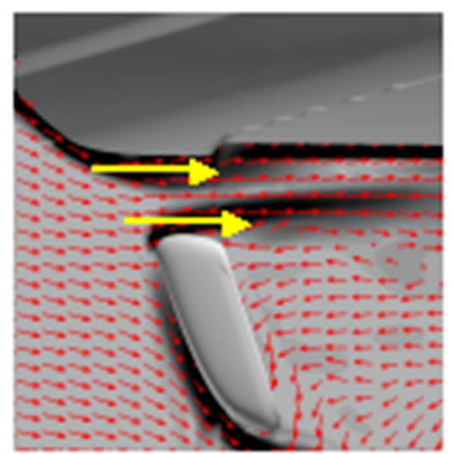

(b) Mirror Model B

Low High

Fig. 12 Distribution of velocity vector and vorticity $\omega_{z}$ (color) the $x y$ section (CFD results)

Fig. 12 shows the time-averaged distribution of velocity vector and $z$ direction vorticity $\omega_{z}$ in the $x y$ section. In Mirror Model A, the separation vortex from front pillar interacts with that from sideview mirror in the space between the mirror and vehicle body. On the other hand, no interaction between the separation vortices from front pillar and mirror is observed in Mirror Model B. 
To investigate the behavior of these separation vortices in detail, the time variation of vorticity $\omega_{z}$ is shown in Fig. 13. In Mirror Model A, the separation vortex coming from front pillar interacts with that from the inside of mirror, which results in the strong vorticity fluctuation (A region in Fig. 13). It indicates that the quadruple acoustic source exists in this spatial domain. In addition, Karman vortex (B and $\mathrm{C}$ regions in Fig. 13) periodically discharged from the sideview mirror enters into the boundary layer (B region in Fig. 13) developed from front pillar, which strengthens the instability of boundary layer. On the other hand, the separation vortex from front pillar is almost parallel to that from sideview mirror in Mirror Model B, and the change of vorticity is small. Moreover, the variation of Karman vortex that occurs from the sideview mirror is also small.
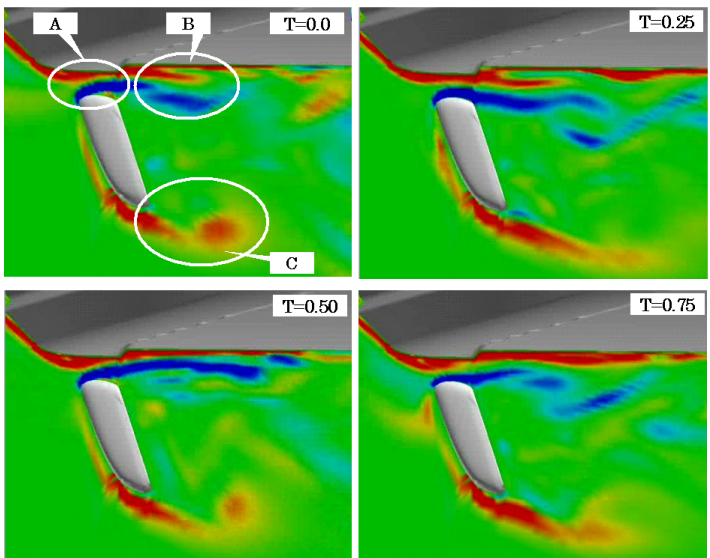

(a) Mirror Model A
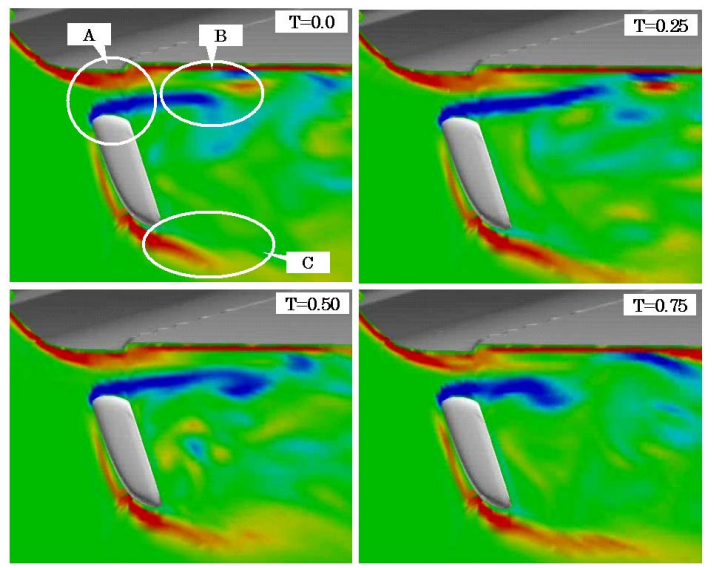

(b) Mirror Model B

Fig. 13 Time variation of vorticity $\left(\omega_{z}\right)$ (CFD results)

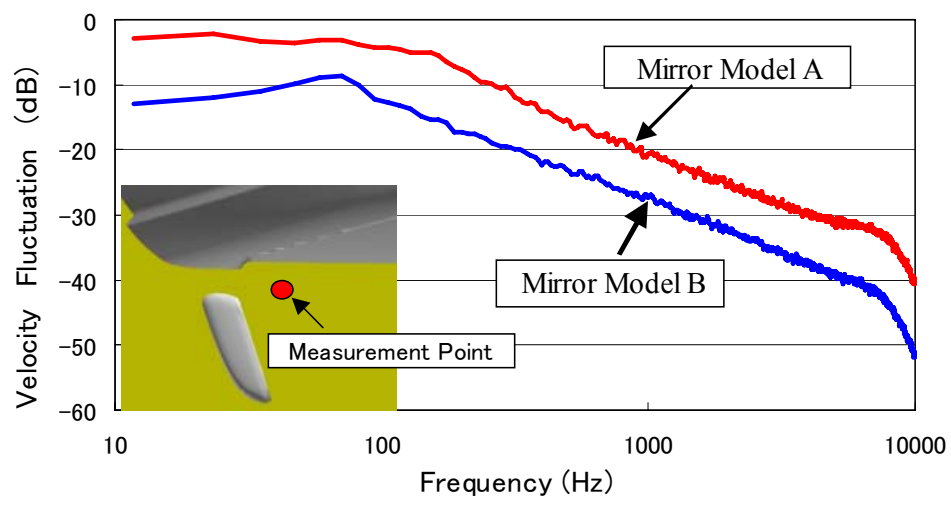

Fig. 14 Spectrum of velocity fluctuations (Experiment results) 
Meanwhile, to confirm the fluctuation of such flow field, Fig. 14 shows the spectrum of velocity fluctuation measured by wind tunnel experiment in the surrounding of side window. The velocity fluctuation of Mirror Model B becomes significantly small compared with that of Mirror Model A. This agrees to the variation of vorticity shown in Fig. 13, and is able to support the simulation results.

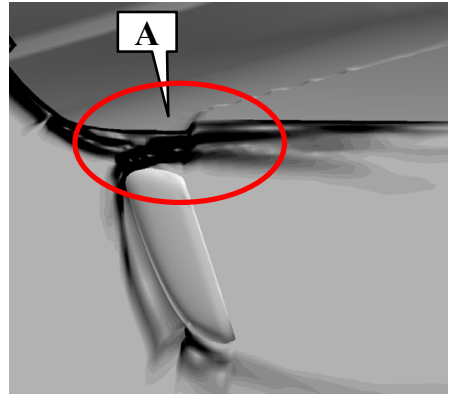

(a) Mirror Model A

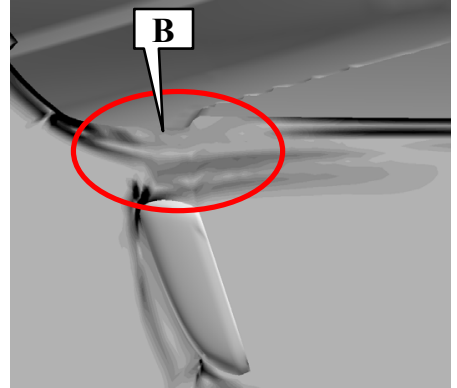

(b) Mirror Model B

Low

High

Fig. 15 Distribution of Powell's acoustic source on $x y$ section (CFD Results)

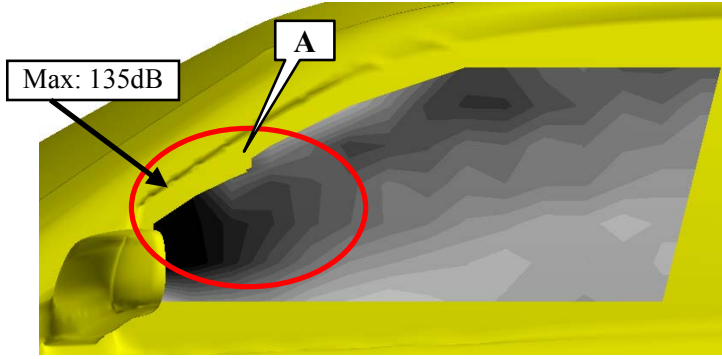

(a) Mirror Model A

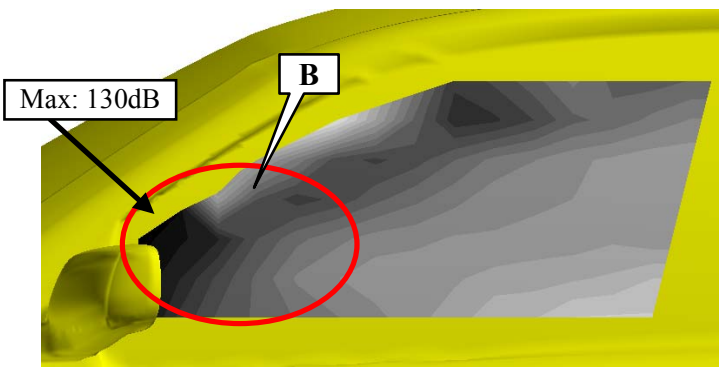

(b) Mirror Model B

$120 \mathrm{~dB}$

$130 \mathrm{~dB}$

Fig.16 Distribution of pressure fluctuation on side window (Experiment Results)

Next, the relation between flow field and aerodynamic noise generation was investigated. Fig. 15 shows the Powell's acoustic source Sp obtained by CFD.

$$
S_{p}=(\nabla \bullet(\omega \times u))
$$

Powell's acoustic source $S_{p}$ shows that aerodynamic noise is generated by the time variation and spatial variation of vorticity. Fig. 16 shows the distribution of pressure fluctuation on side window measured by experiment. In Mirror Model A of Fig. 15(a), the 
dark color in A region shows that Powell's acoustic source is strong, and quadruple aerodynamic noise source $※$ is observed. On the other hand, in A region of Fig. 16(a) measured by experiment, the pressure fluctuation on the surface of side window behind sideview mirror base is strong, which demonstrates the aerodynamic noise is generated by dipole acoustic source. Furthermore, compared with Mirror Model A, both Powell's acoustic source (B region in Fig. 15(b)) and pressure fluctuation on side window (B region in Fig. 16(b)) become small in Mirror Model B, and the acoustic source of aerodynamic noise is reduced.

(※ In this paper, the acoustic source on the space is expressed as 'quadruple', and the sound source on the surface of vehicle is expressed as 'dipole'. Although the Powell's acoustic source is shown as dipole type, the Lighthill's aerodynamic theory shows the sound source is shown in the form of quadruple. The acoustic source on the side window neighborhood space doesn't satisfy all characteristics of quadruple (For instance, it is proportional to the eighth power of the vehicle speed etc.), and some characteristics of dipole is also included. )

The reduction of quadruple aerodynamic noise generated by the interaction of separation vortices from front pillar and sideview mirror, finally appears to the reduction of dipole aerodynamic noise by means of pressure fluctuation in side window surface behind sideview mirror. Although the contribution of both acoustic sources cannot be separated, it is shown that these acoustic sources are attributed to the above-mentioned instability of boundary layer.

\section{Conclusions}

In this study, the mechanism of aerodynamic noise generated from the front pillar and sideview mirror in production vehicle has been clarified by applying wind tunnel experiment and CFD. The following conclusions are obtained.

The aerodynamic noise is generated by the fluctuation of separated flow that develops from front pillar and sideview mirror. The fluctuation is induced by the following two reasons:

(1) The interaction of flow separation vortices from front pillar and sideview mirror strengths the velocity fluctuation of separated flow.

(2) The Karman vortex occurred from the sideview mirror affect the separated flow developed from front pillar, and make it unstable further.

To reduce the aerodynamic noise, it is important to control the interaction of flow separation vortices generated from front pillar and sideview mirror, and inhibit the variation of Karman vortex coming from sideview mirror. So it is necessary to optimize the front pillar shape, sideview mirror shape and the position related to the reduction of the aerodynamic noise.

\section{References}

(1) Lighthill M. J., "On sound generated aerodynamically: I. General theory", Proc. R. Soc. Lond. A 211 (1952), pp. 564-587.

(2) Powell A., Theory of vortex sound, J. Acoust. Soc. Am. 33 (1964), pp. 177-195.

(3) Curle N., The influence of solid boundaries upon aerodynamics sound. Proc. R. Soc. Lond. A 231 (1955), pp. 504-514.

(4) Alam F., Watkins S., Zimmer G. and Humphris C., Effects of Vehicle A-pillar Shape on Local Mean and Time-Varying Flow Properties, Society of Automotive Engineers, 2001-01-1086 (2001).

(5) Kobayashi, T, Nouzawa,T, Design and Aerodynamic Engineering of Vehicle, Asakura Publishing, (1998), pp. 113-140. (in Japanese)

(6) Hashimoto, T., Hashiguchi, M., Nouzawa, T., and Miyamoto, T., Mazda Wind Tunnel, Journal of the Society of Automotive Engineers, Vol.39 (1985), pp.822-826. (in Japanese) 
(7) Kawamura T. and Kuwahara K., Computation of High Reynolds Number Flow around a Circular Cylinder with Surface Roughness, American Institute of Aeronautics and Astronautics, AIAA-84-0340, (1984).

(8) Tsuboi K., Shirayama S., Oana M. and Kuwahara K., Computational Study of the Effect of Base Slant, Second International Conference on Supercomputing in the Automotive Industry, (1988).

(9) Li Y., Kasaki N., Tsunoda H., Nakamura T. and Nouzawa T., Evaluation of Wind Noise Sources Using Experimental and Computational Methods, Society of Automotive Engineers, 2006-01-0343 (2006). 